\section{Incidence and risk factors of}

\section{vancomycin-associated acute}

\section{kidney injury in a single center: Retrospective study}

\author{
Tanuja Yalamarti ${ }^{1}$, Shahrzad Zonoozi ${ }^{1 *}$, Kwabena Adu Ntoso ${ }^{2}$ \\ and Pooneh Alborzi ${ }^{2}$
}

${ }^{1}$ Pennsylvania Hospital, USA

${ }^{2}$ Pennsylvania Hospital, Renal, Electrolyte and Hypertension, USA

\section{Abstract}

Background: There is enough evidence to suggest that vancomycin increases the risk of acute kidney injury (AKI) but the exact mechanism is not well understood. This study aims to understand the incidence of vancomycin-associated acute kidney injury (VA-AKI) among hospitalized patients and to identify the risk factors for VA-AKI.

Methods: Patients aged 18 and above who received a minimum of 24 hours of intravenous vancomycin and who had serial creatinine measurements over a 13-month period were identified through electronic records. Patients with pre-existing AKI, or eGFR of less than $30 \mathrm{ml} / \mathrm{min}$, and patients with end stage kidney disease were excluded. Results were analyzed using t-test and Fisher's test. A logistic regression model was used to identify the predictors for VA-AKI.

Results: From the 598 patients who met the inclusion criteria, 70 developed AKI. Compared to those without AKI, patients with VA-AKI had higher mean serum vancomycin trough levels $(22.6$ $\mathrm{mg} / \mathrm{L}$ vs. $14.6 \mathrm{mg} / \mathrm{L}$ ), and a statistically significant longer duration of vancomycin use (6.7 vs. 5.2 days). Multivariate analysis revealed that serum vancomycin level of $>20 \mathrm{mg} / \mathrm{L}$ was associated with a six-fold increase in odds of VA-AKI when compared to those with vancomycin levels $<15$ $\mathrm{mg} / \mathrm{L}$. The presence of hypotension, iodinated contrast use, and concomitant use of piperacillintazobactam were all associated with increased odds of VA-AKI.

Conclusion: The incidence of VA-AKI in hospitalized patients with eGFR $>30 \mathrm{ml} / \mathrm{min}$ was $11.7 \%$. Serum vancomycin levels of $>20 \mathrm{mg} / \mathrm{L}$, hypotension and administration of iodinated contrast significantly increased the risk of VA-AKI. Piperacillin-tazobactam, when used with vancomycin, was noted to be an independent predictor of AKI, regardless of serum vancomycin trough levels, prompting a reevaluation of the safety of this widespread practice as empiric therapy. Close monitoring of kidney function, avoiding high serum vancomycin levels, maintaining hemodynamic stability, and avoiding unnecessary use of iodinated contrast seem to be essential for the prevention of VA-AKI.
More Information

*Address for Correspondence: Shahrzad Zonoozi, Department of Medicine, University of Pennsylvania Hospital, USA Email: shahrzadz@gmail.com

Submitted: January 04, 2021

Approved: March 05, 2021

Published: March 08, 2021

How to cite this article: Yalamarti T, Zonoozi S, Adu Ntoso K. Alborzi P. Incidence and risk factors of vancomycin-associated acute kidney injury in a single center: Retrospective study. J Clini Nephrol. 2021; 5: 010-016.

DOI: 10.29328/journal.jcn.1001067

Copyright: (c) 2021 Yalamarti T, et al. This is an open access article distributed under the Creative Commons Attribution License, which permits unrestricted use, distribution, and reproduction in any medium, provided the original work is properly cited.

Check for updates

OPEN ACCESS

\section{Introduction}

Vancomycin is a glycopeptide antibiotic widely used for the management of methicillin-resistant Staphylococcus aureus (MRSA) infections. Earlier preparations of vancomycin in the 1950s were relatively impure and, as a result, was referred to as "Mississippi Mud". Previous reports showed that vancomycin could be both nephrotoxic and ototoxic [1]. Despite improvements in the purification process, literature suggests that vancomycin is still associated with $5 \%-40 \%$ increased incidence of acute kidney injury (AKI) [2-6]. A recent systematic review and meta-analysis suggested that use of vancomycin has a relative risk of 2.45 for development of AKI [7].

The exact mechanism of AKI with vancomycin use (VAAKI) remains unclear; however, studies suggest that certain risk factors may increase the risk. Obesity $[8,9]$ intensive care unit (ICU) stay [10] higher doses of vancomycin, prolonged duration of treatment, elevated serum vancomycin levels 
[11] and concomitant treatment with nephrotoxic agents [12], have been implicated as risk factors which may have a role in the development of VA-AKI $[2,3,10,13]$. Hirai and colleagues observed that patients in their study showed poor recovery from vancomycin nephrotoxicity although vancomycin trough concentrations improved. In cases where vancomycin was continued, nephrotoxicity recovered in $40 \%$ of the patients [14]. Although VA- AKI is usually considered a reversible phenomenon, the consequences of AKI can be severe, ranging from increased length of hospitalization, long term dialysis, and transplantation.

Other associated risk factors include concomitant treatment with aminoglycoside [15], or piperacillintazobactam [16-18]. We believe that further systematic studies are needed to confirm and quantify the risk of VA-AKI and to identify the potential risk factors for the development of VA-AKI. We have, therefore, conducted a retrospective review of vancomycin use in our hospital and investigated the incidence of VA-AKI, in order to identify risk factors for the development of VA-AKI.

\section{Methods}

\section{Inclusion and exclusion criteria}

This is a retrospective cohort study of patients identified through a query of electronic health records (EHR) at a University-affiliated community hospital. We included all patients 18 years and older admitted between 01/01/2017 and 02/01/2018 who received greater than 24 hours of intravenous (IV) vancomycin. Vancomycin was initiated as deemed appropriate by the clinician for suspected or proven Gram-positive infection, either on admission or during hospital stay, and dosing was adjusted for renal function.

Patients with estimated glomerular filtration rate (eGFR) of less than $30 \mathrm{ml} / \mathrm{min}$ (based on the Modified Diet in Renal Disease [MDRD] equation) including those with end stage renal disease on renal replacement therapy were excluded. Patients with pre-existing AKI prior to vancomycin administration or who had less than four serum creatinine levels during their admission were also excluded.

\section{Covariates}

Data was reviewed from the EHR to extract information on age, body mass index (BMI), admission diagnosis, comorbidities, other medication use, baseline and peak serum creatinine, highest serum trough vancomycin level (or highest serum vancomycin level obtained when true trough not obtained), duration of vancomycin therapy and cumulative dosage, hypotension, admission hemoglobin, acknowledgement of AKI, and renal consultation during admission. Hypotension was defined by mean arterial pressure less than $65 \mathrm{mmHg}$ for more than three consistent readings or when vasopressors were used that admission. Baseline creatinine was the most recent serum creatinine value prior to vancomycin use. History of diabetes mellitus (DM), heart disease (congestive heart failure [CHF], arrhythmia [including supraventricular and ventricular tachycardia, as well as sick sinus syndrome and bradyarrhythmias and coronary artery disease [CAD]) and peripheral vascular disease (PVD) were identified based on chart review of medical notes. The use of antibiotics including amoxicillin-clavulanic acid, cefepime, ceftriaxone, piperacillin-tazobactam, caspofungin, azithromycin, doxycycline, ampicillin, aztreonam, sulfamethoxazoletrimethoprim, clindamycin, metronidazole, oseltamivir, and acyclovir were included. These antibiotics were included when used in combination with IV vancomycin at therapeutic doses adjusted for the renal function for at least 24 hours. Non-steroidal anti-inflammatory drugs (NSAIDs) included ibuprofen, ketorolac, diclofenac and celecoxib. Diuretics included furosemide or other loop diuretics, chlorthalidone, hydrochlorothiazide and spironolactone. Use of angiotensin converting enzyme inhibitor/angiotensin receptor blockade (ACEi/ARB) and iodinated contrast was also included.

\section{Vancomycin Associated Acute kidney injury (VA-AKI)}

AKI was defined per the Kidney Disease Improving Global Outcomes (KDIGO) guideline, which for stage 1 is an increase in serum creatinine of greater than or equal to 0.3 $\mathrm{mg} / \mathrm{dL}$ within 48 hours or an increase in serum creatinine to greater than or equal to 1.5 times baseline within the prior 7 days. Stage 2 AKI is defined as an increase in serum creatinine 2-2.9 times the baseline, and stage 3 is defined as an increase in serum creatinine greater than 3 times the baseline or initiation of renal replacement therapy. All cases who developed AKI were considered VA-AKI as every patient was receiving IV vancomycin.

All medical records, including all nephrology consultation notes, and other notes that documented identification and possible etiology of AKI were reviewed. Final decision regarding the etiology of the AKI was based on nephrology notes when present and/or chart review by the research investigator nephrologist who reviewed the medical records personally.

\section{Endpoints}

The primary endpoint was the incidence of AKI in patients who received vancomycin, defined as VA-AKI. Acute nephrotoxicity solely related to vancomycin use was determined based on the absence of an alternative explanation of the acute kidney injury and on chart review by a nephrologist. Secondary endpoints included identification of risk factors which predispose patients to develop VA-AKI.

\section{Statistical analysis}

Descriptive statistics were conducted on all the variables. Baseline demographics and clinical characteristics were tabulated, mean with standard deviation for continuous 
variables and frequencies (proportions) with percentages for categorical variables. Statistical significance between those who developed VA-AKI and those who did not was obtained by $t$ - test for the continuous variables, and from Fisher's exact test for the categorical variables reported as the $p$-value.

Univariate logistic regression analyses were performed for each exposure variable to compare the group with nephrotoxicity vs. those without, and to identify all the possible risk factors. A multivariate analysis was then constructed to test the association of identified risk factors while controlling the other variables. SAS statistical software (SAS Institute, Cary, NC) was used for statistical analysis.

\section{Results}

Our study was based on the 598 patients who met the inclusion criteria. 70 (11.7\%) patients developed VA-AKI. The cause of AKI in 12 cases (17\% of VA-AKI patients and $2 \%$ of total population) was deemed to be directly related to vancomycin toxicity, as determined by the nephrology consultation notes and/or chart review by the research investigator nephrologist. The cause of AKI in the rest of the population was mainly stated as multifactorial.
In those with VA-AKI, $48.6 \%(n=34)$ had stage $1,21.4 \%$ $(n=15)$ had stage 2 , and $30 \%(n=21)$ had stage 3 AKI. AKI was not documented in the medical records in 28 (40\%) cases based on the changes in the creatinine per KDIGO definition.

Table 1 demonstrates the demographics of patients with and without VA-AKI. There were no significant differences in gender, mean age, and baseline serum creatinine between the two groups. Prevalence of comorbidities including anemia, diabetes mellitus, hypertension, peripheral vascular disease, heart disease, sickle cell disease and malignancy was not statistically different in the two groups.

Compared to those with no AKI, patients with VA-AKI had a longer duration of treatment with vancomycin ( 6.7 vs. 5.2 days) and were noted to have a higher mean serum vancomycin trough level $(22.6 \mathrm{mg} / \mathrm{L} v s .15 \mathrm{mg} / \mathrm{L})$. Those with VA-AKI had a higher mean cumulative vancomycin dose of $13.3 \mathrm{mg} / \mathrm{L}$ compared to those with no AKI who had a mean cumulative vancomycin dose of $11.7 \mathrm{mg} / \mathrm{L}$. However, this association was not statistically significant.

There was a significantly higher incidence of baseline CKD stage 3 (eGFR of less than $60 \mathrm{ml} / \mathrm{min}$ based on the MDRD

Table 1: Comparison of clinical characteristics in VA-AKI and no - AKI patients.

\begin{tabular}{|c|c|c|c|c|}
\hline Variables & All $\left(n=598^{*}\right)$ & No - AKI $(n=528)$ & VA-AKI $\left(n=70^{*}\right)$ & $p$ value \\
\hline Age (mean +/- STD) & $57.3+/-17.5$ & $57.2+/-17.1$ & $58.6+/-19.8$ & 0.5602 \\
\hline Female $(n ; \%)$ & $258 ;(43.1 \%)$ & $221 ;(41.9 \%)$ & $37 ;(52.9 \%)$ & 0.0949 \\
\hline Male $(n ; \%)$ & $340 ;(56.9 \%)$ & $307 ;(58.1 \%)$ & $33 ;(47.1 \%)$ & \\
\hline $\mathrm{BMI}<25(n ; \%)$ & $194 ;(12.1 \%)$ & $164 ;(31.1 \%)$ & $30 ;(42.9 \%)$ & 0.0569 \\
\hline Hemoglobin (g/dL) (mean +/- STD) & $11.6+/-2.4$ & $11.6+/-2.3$ & $11.2+/-2.4$ & 0.1254 \\
\hline Baseline creatinine (mg/dL) (mean +/- STD) & $0.9+/-0.3$ & $0.9+/-0.3$ & $0.8+/-0.4$ & 0.4908 \\
\hline Highest serum vancomycin level (mg/L) (mean +/- STD) & $16.0+/-7.5$ & $15.0+/-6.6$ & $22.6+/-9.5$ & $<0.0001$ \\
\hline Vancomycin cumulative dose (g) (mean +/- STD) & $11.9+/-11.3$ & $11.7+/-11.0$ & $13.3+/-13.7$ & 0.2709 \\
\hline Vancomycin duration in days (mean +/- STD) & $5.4+/-4.4$ & $5.2+/-4.0$ & $6.7+/-6.4$ & 0.0098 \\
\hline \multicolumn{5}{|c|}{ Comorbidities $(n ; \%)$} \\
\hline Anemia $(\mathrm{Hb}<8 \mathrm{~g} / \mathrm{dL})$ & $34 ;(5.7 \%)$ & $30 ;(5.7 \%)$ & $4 ;(5.7 \%)$ & 1.0000 \\
\hline Diabetes & $145 ;(24.2 \%)$ & $127 ;(24.1 \%)$ & $18 ;(25.7 \%)$ & 0.7674 \\
\hline Hypertension & $244 ;(40.8 \%)$ & $214 ;(40.5 \%)$ & $30 ;(42.9 \%)$ & 0.7004 \\
\hline Peripheral vascular disease & $22 ;(3.7 \%)$ & $19 ;(3.6 \%)$ & $3 ;(4.3 \%)$ & 0.7343 \\
\hline Heart disease & $155(25.9 \%)$ & $136 ;(25.8 \%)$ & $19 ;(27.1 \%)$ & 0.7736 \\
\hline Sickle cell disease & $28(4.7 \%)$ & $22 ;(4.2 \%)$ & $6 ;(8.6 \%)$ & 0.1244 \\
\hline Malignancy & $109(18.2 \%)$ & $94 ;(17.8 \%)$ & $15 ;(21.4 \%)$ & 0.5094 \\
\hline \multicolumn{5}{|c|}{ CKD groups $(n ; \%)$} \\
\hline eGFR $\geq 60 \mathrm{ml} / \mathrm{min}$ & $550(92.0 \%)$ & $492 ;(93.2 \%)$ & $58 ;(82.9 \%)$ & 0.0077 \\
\hline eGFR $<60 \mathrm{ml} / \mathrm{min}$ & $48(8 \%)$ & $36 ;(6.8 \%)$ & $12 ;(17.1 \%)$ & \\
\hline \multicolumn{5}{|c|}{ Other variables $(n ; \%)$} \\
\hline Hypotension & $96(16.1 \%)$ & $68 ;(12.9 \%)$ & $28 ;(40.0 \%)$ & $<0.0001$ \\
\hline lodinated contrast & $181(30.3 \%)$ & $149 ;(28.2 \%)$ & $32 ;(45.7 \%)$ & 0.0036 \\
\hline Diuretics & $163(27.3 \%)$ & $137 ;(25.9 \%)$ & $26 ;(37.1 \%)$ & 0.0624 \\
\hline NSAIDs & $175(29.3 \%)$ & $159 ;(30.1 \%)$ & $16 ;(22.9 \%)$ & 0.2632 \\
\hline ACEi/ARB & $101(16.9 \%)$ & $92 ;(17.4 \%)$ & $9 ;(12.9 \%)$ & 0.3987 \\
\hline \multicolumn{5}{|c|}{ Antibiotics $(n ; \%)$} \\
\hline Piperacillin-tazobactam & $189(31.6 \%)$ & $151 ;(28.6 \%)$ & $38 ;(54.3 \%)$ & $<0.0001$ \\
\hline Cefepime & $155(25.9 \%)$ & $131 ;(24.8 \%)$ & $24 ;(34.3 \%)$ & 0.1095 \\
\hline Gentamicin & $46(7.7 \%)$ & $39 ;(7.4 \%)$ & $7 ;(10.0 \%)$ & 0.4713 \\
\hline Metronidazole & $99(16.6 \%)$ & $80 ;(15.2 \%)$ & $19 ;(27.1 \%)$ & 0.0160 \\
\hline
\end{tabular}

Abbreviations: STD: Standard Deviation; Hb: Hemoglobin; eGFR: estimated Glomerular Filtration Rate; NSAIDS: Non-Steroidal Anti-Inflammatory; ACEi/ARB: Angiotensin Converting Enzyme Inhibitor/Angiotensin Receptor Blockade

${ }^{*}$ maximum $n$ in group; for highest serum vancomycin level $n=454,396$ and 58 for all, those with no AKI and those with VA-AKI respectively. 
equation) in those with VA-AKI (17.1\% compared to 6.8\%). Those who developed VA-AKI had a statistically significant higher incidence of hypotension ( $40 \%$ vs. 13\%) and iodinated contrast use (46\% vs. 28\%) compared to those without AKI. Contrast-induced nephropathy was considered the cause of acute kidney injury in 7\% of patients with VA-AKI. There was no statistically significant difference in the use of diuretics, NSAIDs or ACEi/ARBs between the two groups.

Those with VA-AKI had a significantly higher incidence of concomitant use of piperacillin-tazobactam or metronidazole compared to the no AKI group. Total number of concomitant antibiotics was not statistically different in both the groups. We further subdivided those with VA-AKI into stages of AKI. No discernible associations were found when comparing stages of AKI with various risk factors.

The continuous variables are age, hemoglobin, baseline creatinine, highest serum vancomycin level, and vancomycin duration measured in terms of mean/standard duration. The rest of the variables are categorically reported as numbers and percentages.

We further studied the identified risk factors for VA-AKI and adjusted for possible confounding variables in table 2 .

Univariate analysis revealed that serum trough vancomycin levels $>20 \mathrm{mg} / \mathrm{L}$ were associated with sixfold increased odds of VA-AKI when compared to those with highest serum trough vancomycin levels $<15 \mathrm{mg} / \mathrm{L}$ $[6.46(3.33,12.5)]$; this association remained robust even after adjusting for age, gender, comorbidities, diuretic use, iodinated contrast use, hypotension and piperacillintazobactam use $[7.18(3.37,15.3)]$. Duration of vancomycin use only marginally increased the odds of VA-AKI [1.05 $(1.01,1.10)]$. While increased duration of vancomycin use was associated with increased odds of VA-AKI in the univariate analysis, this association was not seen following adjustment.

VA-AKI was seen more frequently in group with eGFR $<60 \mathrm{ml} / \mathrm{min}$, however, this association was not significant when adjusted for other variables.

Hypotension was associated with a four-fold increase in odds of VA-AKI in our population [4.51 $(2.62,7.75)]$, which remained robust after adjustment for variables as above [4.55 $(2.46,8.42)]$. Iodinated contrast use was associated with two-fold increased odds of VA-AKI $[2.14(1.29,3.56)]$. This association remained but was attenuated [1.82 (1.03, 3.21)] following adjustment for age, gender, comorbidities, diuretic use, iodinated contrast, hypotension, and highest serum trough vancomycin level $>20 \mathrm{mg} / \mathrm{L}$.

Analysis of concomitant antimicrobial use revealed

Table 2: Predictors for VA-AKI.

\begin{tabular}{|c|c|c|c|c|c|}
\hline & & Univariate & Model 1 & Model 2 & Model 3 \\
\hline \multicolumn{6}{|l|}{ Variable } \\
\hline Age & $>65$ vs. $18-65$ & $1.25(0.75,2.08)$ & $1.23(0.67,2.23)$ & $1.09(0.58,2.04)$ & $1.09(0.57,2.07)$ \\
\hline Sex & Male vs. Female & $0.64(0.39,1.06)$ & $0.63(0.37,1.06)$ & $0.56(0.32,0.96)$ & $0.58(0.33,1.02)$ \\
\hline $\mathrm{BMI}<25$ & Yes, vs. No & $1.66(1.00,2.77)$ & $1.50(0.85,2.64)$ & $1.26(0.70,2.28)$ & $1.51(0.82,2.76)$ \\
\hline Baseline creatinine $(\mathrm{mg} / \mathrm{dL})$ & & $0.72(0.29,1.81)$ & $0.89(0.33,2.37)$ & $1.01(0.37,2.75)$ & $0.61(0.22,1.70)$ \\
\hline Vancomycin duration (days) & & $1.05(1.01,1.10)$ & $1.04(1.00,1.09)$ & $1.03(0.98,1.08)$ & $0.98(0.93,1.04)$ \\
\hline Vancomycin cumulative dose (g) & & $1.01(0.99,1.03)$ & $1.01(0.99,1.03)$ & $1.00(0.98,1.02)$ & $0.98(0.96,1.01)$ \\
\hline Highest serum vancomycin level & $15-20$ vs. $<15$ & $0.89(0.37,2.16)$ & $0.95(0.38,2.38)$ & $0.97(0.38,2.46)$ & $0.92(0.37,2.31)$ \\
\hline$(\mathrm{mg} / \mathrm{L})$ & $>20$ vs. $<15$ & $6.46(3.33,12.5)$ & $7.32(3.57,15.0)$ & $7.18(3.37,15.3)$ & $7.13(3.40,14.9)$ \\
\hline Anemia $(\mathrm{Hb}<8 \mathrm{~g} / \mathrm{dL})$ & Yes, vs. No & $1.01(0.34,2.95)$ & $0.77(0.23,2.52)$ & $0.70(0.20,2.45)$ & $0.62(0.16,2.33)$ \\
\hline Diabetes & Yes, vs. No & $1.09(0.62,1.94)$ & $1.05(0.57,1.96)$ & $1.27(0.66,2.42)$ & $1.00(0.50,1.97)$ \\
\hline Hypertension & Yes, vs. No & $1.10(0.66,1.82)$ & $1.18(0.67,2.07)$ & $1.40(0.77,2.55)$ & $1.64(0.87,3.08)$ \\
\hline Peripheral vascular disease & Yes, vs. No & $1.20(0.35,4.16)$ & $1.00(0.27,3.67)$ & $1.22(0.31,4.74)$ & $1.45(0.37,5.74)$ \\
\hline Heart disease & Yes, vs. No & $1.07(0.61,1.88)$ & $1.11(0.58,2.09)$ & $0.77(0.37,1.58)$ & $0.73(0.35,1.52)$ \\
\hline Sickle cell disease & Yes, vs. No & $2.16(0.84,5.52)$ & $2.13(0.71,6.36)$ & $2.00(0.63,6.37)$ & $2.41(0.71,8.13)$ \\
\hline Malignancy & Yes, vs. No & $1.26(0.68,2.32)$ & $1.32(0.69,2.54)$ & $1.11(0.56,2.17)$ & $0.96(0.48,1.94)$ \\
\hline eGFR (ml/min) & $\geq 60$ vs. $<60$ & $0.35(0.17,0.72)$ & $0.38(0.18,0.81)$ & $0.43(0.19,1.00)$ & $0.57(0.24,1.34)$ \\
\hline Hypotension & Yes, vs. No & $4.51(2.62,7.75)$ & $4.80(2.64,8.72)$ & $4.55(2.46,8.42)$ & $4.47(2.36,8.46)$ \\
\hline lodinated contrast & Yes, vs. No & $2.14(1.29,3.56)$ & $1.85(1.08,3.14)$ & $1.77(1.02,3.08)$ & $1.82(1.03,3.21)$ \\
\hline Diuretics & Yes, vs. No & $1.69(1.00,2.84)$ & $1.71(0.94,3.13)$ & $1.19(0.63,2.27)$ & $1.01(0.52,2.00)$ \\
\hline NSAIDs & Yes, vs. No & $0.69(0.38,1.24)$ & $0.61(0.32,1.16)$ & $0.65(0.34,1.26)$ & $0.63(0.32,1.25)$ \\
\hline ACEi/ARB & Yes, vs. No & $0.70(0.34,1.46)$ & $0.66(0.30,1.47)$ & $0.74(0.32,1.72)$ & $0.80(0.33,1.92)$ \\
\hline Piperacillin-tazobactam & Yes, vs. No & $2.96(1.79,4.92)$ & $3.01(1.78,5.09)$ & $2.50(1.44,4.35)$ & $2.11(1.18,3.75)$ \\
\hline Cefepime & Yes, vs. No & $1.58(0.93,2.69)$ & $1.68(0.96,2.96)$ & $1.46(0.81,2.63)$ & $1.09(0.58,2.02)$ \\
\hline Gentamicin & Yes, vs. No & $1.39(0.60,3.25)$ & $1.68(0.69,4.06)$ & $1.83(0.70,4.76)$ & $2.13(0.79,5.72)$ \\
\hline Metronidazole & Yes, vs. No & $2.09(1.17,3.72)$ & $2.05(1.11,3.79)$ & $1.84(0.96,3.53)$ & $1.65(0.84,3.24)$ \\
\hline \multirow{2}{*}{ Number of concomitant antibiotics } & 2 vs. 1 & $1.86(0.97,3.57)$ & $1.59(0.81,3.13)$ & $1.81(0.89,3.66)$ & $1.78(0.87,3.66)$ \\
\hline & $\geq 3$ vs. 1 & $1.87(0.98,3.57)$ & $1.49(0.75,2.95)$ & $1.42(0.70,2.90)$ & $1.49(0.72,3.08)$ \\
\hline
\end{tabular}

Model 1: Adjusted for age, gender, comorbidities, and piperacillin-tazobactam use

Model 2: Adjusted for age, gender, comorbidities, diuretic use, iodinated contrast, hypotension, and piperacillin-tazobactam use

Model 3: Adjusted for age, gender, comorbidities, diuretic use, iodinated contrast, hypotension, and highest serum vancomycin trough level > 20 mg/L.

Abbreviations: STD: Standard Deviation; Hb: Hemoglobin; eGFR: estimated Glomerular Filtration Rate; NSAIDS: Non-Steroidal Anti-Inflammatory; ACEi/ARB: Angiotensin

Converting Enzyme Inhibitor/Angiotensin Receptor Blockade 
that piperacillin-tazobactam and metronidazole use was associated with increased odds of VA-AKI $[2.96(1.79,4.92)$ and 2.09 (1.17, 3.72) respectively]. Following adjustment for above variables, piperacillin-tazobactam continued to be associated with a two-fold increased odds of VA-AKI $[2.11(1.18,3.75)]$ while metronidazole use was no longer significantly associated with increased odds of VA-AKI [1.65 $(0.84,3.24)]$.

Data are expressed as odds ratio (95\% confidence interval).

\section{Discussion}

Vancomycin is often used as a first line empiric therapy for many moderate-to-severe gram-positive infections and has been associated with AKI [4]. Given the widespread use of vancomycin, there are reports of VA-AKI ranging between $5 \%$ to $40 \%[2-6]$.

In our study, $11.7 \%$ of patients who received vancomycin and met the inclusion criteria developed VA-AKI. Only in 2\% of the total 598 cohort patients was vancomycin considered the most likely cause. In $83 \%$ of cases of VA-AKI, the cause of AKI was determined as multifactorial with vancomycin being a contributing factor. In our study, patients with high vancomycin levels had a significantly higher incidence of AKI. This finding suggests that even when vancomycin was not considered the main cause of AKI, it could have played a role in the development of VA-AKI.

We observed significant differences in the serum trough vancomycin levels in the groups with VA-AKI compared to those without AKI (mean level of $22.6 \mathrm{mg} / \mathrm{L} \mathrm{vs.} 15 \mathrm{mg} / \mathrm{L}$ respectively; $p<0.001$ ). In our study, odds of VA-AKI was significantly increased in the group with serum vancomycin levels greater than $20 \mathrm{mg} / \mathrm{L}$ with odds ratio of 6.46 (3.33, 12.5). This observation remained robust after adjustment for other major risk factors such as hypotension. This finding is of particular importance as it suggests that the association seen between high serum vancomycin trough levels and VAAKI is not confounded by severity of sepsis and hemodynamic instability. A recent study has also shown similar findings [3].

In a meta-analysis by Hal, et al. [6], odds of nephrotoxicity remained increased, at 3.12 (95\% CI, 1.81-5.37; $p<0.01$ ) for patients attaining initial trough levels of $\geq 15 \mathrm{mg} / \mathrm{L}$, indicating that an augmented risk of toxicity occurs in individuals with troughs of $\geq 15 \mathrm{mg} / \mathrm{L}$. In our study, we did not find a higher risk of AKI when highest vancomycin levels were between $15-20 \mathrm{mg} / \mathrm{L}$ compared to those with levels $<15 \mathrm{mg} / \mathrm{L}$ despite adjustment for other variables. Due to the small demarcation in trough values between $15-20 \mathrm{mg} / \mathrm{L}$ and $>20 \mathrm{mg} / \mathrm{L}$, it is possible that vancomycin trough values of $>20 \mathrm{mg} / \mathrm{L}$ could be the main factor driving nephrotoxicity in the $>15 \mathrm{mg} / \mathrm{L}$ strata in prior observational studies. It is also possible that our sample size did not have the power to detect differences at that range.
Earlier studies reported that treatment with vancomycin for a duration of $\geq 14$ days is associated with a higher risk of VA-AKI, defined as a rise in serum creatinine of $\geq 0.5 \mathrm{mg} / \mathrm{dL}$ [19]. However, in our study, odds of VA-AKI was not increased by increased duration of vancomycin use; this is similar to a recent study showing AKI to be associated with high vancomycin trough level rather than dose or duration [20].

The mechanism of vancomycin induced nephrotoxicity is unclear. Several possible mechanisms of injury have been proposed. These include acute tubular injury and/or acute tubulointerstitial nephritis as a result of the induction of oxidative stress, complement activation with inflammatory injury, and mitochondrial damage [21]. Obstructing tubular cast formation has also been proposed as a novel possible cause of VA-AKI [22]. The multifactorial nature of VA-AKI is best confirmed by the findings of two randomized clinical trials which also support the notion that certain clinical factors can augment the severity of the nephrotoxicity [23]. One study [12] conferred that a higher number of nephrotoxic agents used with vancomycin predisposes to increased risk of AKI. Another study even argued that intravenous vancomycin at therapeutic levels as monotherapy may not be nephrotoxic when compared to other antibiotics [24].

Based on the above proposed mechanisms, our study has investigated possible risk factors for the development of VA-AKI. According to our study, the presence of hypotension was a strong and independent predictor of VA-AKI, following adjustment for variables that could be potential confounders. In our population, those with VA-AKI had a statistically significant higher incidence of hypotension when compared to those without AKI: $40 \%$ vs. $13 \%$ respectively. Of those with hypotension and VA-AKI, 36\% of cases of AKI (stages 1 and 2) were not acknowledged in the medical notes by the providers. This pattern was mainly seen in patients who had a creatinine rise which remained within the normal limits set by the EHR (in our system $0.44-1.03 \mathrm{mg} / \mathrm{dL}$ ).

Similarly, simultaneous iodinated contrast use also was associated with increased risk of AKI. The exact mechanism of renal injury with vancomycin in the presence of hypotension or iodinated contrast remains unclear. It is likely that the combination of vancomycin and hypotension or iodinated contrast augments the prerenal state leading to impaired renal perfusion, increasing the risk of oxidative stress and tubular necrosis. This may indicate that, in patients who receive vancomycin, special attention needs to be considered for maintaining hemodynamic stability and preventing hypotension while also trying to avoid iodinated contrast use if possible. The findings from this study do not however prove a causal relationship between vancomycin use in hypotension and AKI. It merely could be that critically ill patients are more likely to receive vancomycin.

In our analysis, increased odds of VA-AKI were not 
observed with concomitant NSAID and vancomycin use although NSAIDs do cause afferent renal artery vasoconstriction. It is possible that the doses of NSAIDs received were not significant or given for a prolonged period to impact the renal function.

Several studies and a systematic review have reported an association between use of concomitant piperacillintazobactam and VA-AKI by mechanisms not well understood [16-18]. Decreased clearance of vancomycin by piperacillintazobactam, resulting in vancomycin accumulation, sometimes muted the impact of vancomycin trough concentrations on AKI [18,25-27]. In our study, we tested a wide variety of concomitant antibiotic usage and found that piperacillin-tazobactam use, as an independent variable, was associated with a significant increase in the risk of VA-AKI. The effect of piperacillin-tazobactam in increasing the risk of AKI remained significant even after adjustment for serum vancomycin levels $>20 \mathrm{mg} / \mathrm{L}$. These results provide an impetus to obtain further insight into the true mechanisms at a molecular level to explain this nephrotoxic effect of vancomycin and piperacillin-tazobactam combination. Although simultaneous use of aminoglycosides with vancomycin is a well-known risk factor for AKI, the association was not observed in our study $[15,24]$. This could perhaps be due to the limited number of patients (46 out of 598 patients) who received this combination.

Our study adds to the literature by looking at a more comprehensive range of variables including administration of a variety of concomitant antibiotics to identify potential risk factors for VA-AKI. Furthermore, the adjustment models for confounders were built to narrow down the risk factors which play a key contributing role in VA-AKI. Identification of such key risk factors helps to build risk prediction models to prescribe and use vancomycin safely, such as in a study conducted by Miyai and colleagues [28].

Although our study contributes to better understanding of the incidence and risk factors of VA-AKI, it does have some limitations. It is a retrospective study and, therefore, any conclusions should be interpreted as exploratory in nature. Furthermore, we were limited by the data that was already collected in the database. As such, there may still be residual confounding effects that we could not ascertain. For example, the significance of concomitant metronidazole use in patients was not persistent once adjusted for other variables, and the significance of this is not clear to us. In order to minimize system errors, however, a detailed chart review of patient records who developed AKI was carried out by a nephrologist once the data was collected. Furthermore, the lack of confirmed trough serum vancomycin levels is a limitation of our study, however, this is more reflective of real-life practices when using vancomycin. Exclusion of patients with eGFR less than 30 is also a limitation of our study.

\section{Conclusion}

The incidence of VA-AKI in hospitalized patients with eGFR greater than $30 \mathrm{ml} / \mathrm{min}$ was $11.7 \%$, and cause of the AKI in most cases was considered as multifactorial. Higher vancomycin levels of more than $20 \mathrm{mg} / \mathrm{L}$ significantly increased the risk of AKI. The presence of hypotension and the administration of iodinated contrast were among other risk factors. Patients with eGFR $<60 \mathrm{ml} / \mathrm{min}$ are at a higher risk of developing VA-AKI. Piperacillin-tazobactam, when used with vancomycin, was noted to be an independent predictor of AKI, regardless of serum vancomycin trough levels, prompting a reevaluation of the safety of the widespread practice of this combination as an empiric therapy. Close monitoring of kidney function, avoiding high serum vancomycin levels, maintaining hemodynamic stability, and avoiding unnecessary use of iodinated contrast seem to be essential for the diagnosis and prevention of VA-AKI.

\section{References}

1. Moellering RC, Jr. Vancomycin: a 50-year reassessment. Clin Infect Dis. 2006; 42: S3-4.

PubMed: https://pubmed.ncbi.nlm.nih.gov/16323117/

2. Elyasi S, Khalili H, Dashti-Khavidaki S, Mohammadpour A. Vancomycin-induced nephrotoxicity: mechanism, incidence, risk factors and special populations. A literature review. Eur J Clin Pharmacol. 2012; 68: 1243-1255.

PubMed: https://pubmed.ncbi.nlm.nih.gov/22411630/

3. Gyamlani G, Potukuchi PK, Thomas F, Akbilgic O, Soohoo M, et al. Vancomycin-Associated Acute Kidney Injury in a Large Veteran Population. Am J Nephrol. 2019; 49: 133-142. PubMed: https://pubmed.ncbi.nlm.nih.gov/30677750/

4. Jorgensen SCJ, Murray KP, Lagnf AM, Melvin S, Bhatia S, et al. A Multicenter Evaluation of Vancomycin-Associated Acute Kidney Injury in Hospitalized Patients with Acute Bacterial Skin and Skin Structure Infections. Infect Dis Ther. 2020; 9: 89-106.

PubMed: https://pubmed.ncbi.nIm.nih.gov/31983021/

5. Qin X, Tsoi MF, Zhao X, Zhang L, Qi Z, et al. Vancomycin-associated acute kidney injury in Hong Kong in 2012-2016. BMC Nephrol. 2020; 21: 41.

6. van Hal SJ, Paterson DL, Lodise TP. Systematic review and metaanalysis of vancomycin-induced nephrotoxicity associated with dosing schedules that maintain troughs between 15 and 20 milligrams per liter. Antimicrob Agents Chemother. 2013; 57: 734-744. PubMed: https://pubmed.ncbi.nlm.nih.gov/23165462/

7. Ray AS, Haikal A, Hammoud KA, Yu AS. Vancomycin and the Risk of AKI: A Systematic Review and Meta-Analysis. Clin J Am Soc Nephrol. 2016; 11: 2132-2140.

PubMed: https://pubmed.ncbi.nlm.nih.gov/27895134/

8. Lodise TP, Lomaestro B, Graves J, Drusano GL. Larger vancomycin doses (at least four grams per day) are associated with an increased incidence of nephrotoxicity. Antimicrob Agents Chemother. 2008; 52 : 1330-1336.

PubMed: https://pubmed.ncbi.nlm.nih.gov/18227177/

9. Rutter WC, Hall RG, Burgess DS. Impact of total body weight on rate of acute kidney injury in patients treated with piperacillin-tazobactam and vancomycin. Am J Health Syst Pharm. 2019; 76: 1211-1217. PubMed: https://pubmed.ncbi.nlm.nih.gov/31369116/

10. Aljefri DM, Avedissian SN, Rhodes NJ, Postelnick MJ, Nguyen K, et al. 
Vancomycin Area Under the Curve and Acute Kidney Injury: A Metaanalysis. Clin Infect Dis. 2019; 69: 1881-1887.

PubMed: https://pubmed.ncbi.nlm.nih.gov/30715208/

11. Bellos I, Daskalakis G, Pergialiotis V. Relationship of vancomycin trough levels with acute kidney injury risk: an exposure-toxicity metaanalysis. J Antimicrob Chemother. 2020; 75: 2725-2734.

PubMed: https://pubmed.ncbi.nlm.nih.gov/32417905/

12. Ueki $T$, Sanematsu E, Furuya $Y$, Shinohara $Y$, Murakami $Y$, et al. Relationship between vancomycin-associated nephrotoxicity and the numberofcombinednephrotoxicagents. Pharmazie. 2020;75:279-283. PubMed: https://pubmed.ncbi.nlm.nih.gov/32539926/

13. Cappelletty D, Jablonski A, Jung R. Risk factors for acute kidney injury in adult patients receiving vancomycin. Clin Drug Investig. 2014; 34: 189-193.

PubMed: https://pubmed.ncbi.nlm.nih.gov/24385282/

14. Hirai T, Hanada K, Kanno A, Akashi M, Itoh T. Risk factors for vancomycin nephrotoxicity and time course of renal function during vancomycin treatment. Eur J Clin Pharmacol. 2019; 75: 859-866. PubMed: https://pubmed.ncbi.nlm.nih.gov/30770940/

15. Farber BF, Moellering RC, Jr. Retrospective study of the toxicity of preparations of vancomycin from 1974 to 1981. Antimicrob Agents Chemother. 1983; 23: 138-141.

PubMed: https://pubmed.ncbi.nlm.nih.gov/6219616/

16. Ciarambino T, Giannico OV, Campanile A, Tirelli P, Para O, et al. Acute kidney injury and vancomycin/piperacillin/tazobactam in adult patients: a systematic review. Intern Emerg Med. 2020; 15: 327-331. PubMed: https://pubmed.ncbi.nlm.nih.gov/32040830/

17. Contejean A, Tisseyre M, Canouï E, Treluyer JM, Kerneis S, et al. Combination of vancomycin plus piperacillin and risk of acute kidney injury: a worldwide pharmacovigilance database analysis. J Antimicrob Chemother. 2021; dkab003.

PubMed: https://pubmed.ncbi.nlm.nih.gov/33617641/

18. Luther MK, Timbrook TT, Caffrey AR, Dosa D, Lodise TP, et al. Vancomycin Plus Piperacillin-Tazobactam and Acute Kidney Injury in Adults: A Systematic Review and Meta-Analysis. Crit Care Med. 2018; 46: 12-20.

PubMed: https://pubmed.ncbi.nlm.nih.gov/29088001/

19. Jeffres MN, Isakow W, Doherty JA, Micek ST, Kollef MH. A retrospective analysis of possible renal toxicity associated with vancomycin in patients with health care-associated methicillin-resistant
Staphylococcus aureus pneumonia. Clin Ther. 2007; 29: 1107-1115. PubMed: https://pubmed.ncbi.nlm.nih.gov/17692725/

20. Hays WB, Tillman E. Vancomycin-Associated Acute Kidney Injury in Critically III Adolescent and Young Adult Patients. J Pharm Pract. 2020; 33: 749-753.

PubMed: https://pubmed.ncbi.nlm.nih.gov/30808269/

21. Perazella MA. Drug-induced acute kidney injury: diverse mechanisms of tubular injury. Curr Opin Crit Care. 2019; 25: 550-557. PubMed: https://pubmed.ncbi.nIm.nih.gov/31483318/

22. Luque $\mathrm{Y}$, Louis $\mathrm{K}$, Jouanneau C, Placier S, Esteve E, et al. Vancomycin-Associated Cast Nephropathy. J Am Soc Nephrol. 2017; 28: 1723-1728.

PubMed: https://pubmed.ncbi.nIm.nih.gov/28082518/

23. Corrado ML. Integrated safety summary of CANVAS 1 and 2 trials: Phase III, randomized, double-blind studies evaluating ceftaroline fosamil for the treatment of patients with complicated skin and skin structure infections. J Antimicrob Chemother. 2010; 65: iv67-iv71. PubMed: https://pubmed.ncbi.nlm.nih.gov/21115456/

24. Gaggl M, Pate V, Sturmer T, Kshirsagar AV, Layton JB. The comparative risk of acute kidney injury of vancomycin relative to other common antibiotics. Sci Rep. 2020; 10: 17282. PubMed: https://pubmed.ncbi.nlm.nih.gov/33057027/

25. Filippone EJ, Kraft WK, Farber JL. The Nephrotoxicity of Vancomycin. Clin Pharmacol Ther. 2017; 102: 459-469.

PubMed: https://pubmed.ncbi.nlm.nih.gov/28474732/

26. Navalkele B, Pogue JM, Karino S, Nishan B, Salim M, et al. Risk of Acute Kidney Injury in Patients on Concomitant Vancomycin and Piperacillin-Tazobactam Compared to Those on Vancomycin and Cefepime. Clin Infect Dis. 2017; 64: 116-123. PubMed: https://pubmed.ncbi.nlm.nih.gov/27986669/

27. Sazanami K, Inose R, Dote S, Horiuchi N, Kobayashi $\mathrm{Y}$, et al. Combination therapy of vancomycin and piperacillin/tazobactam in adult febrile neutropenia patients with haematopoietic malignancies increases the risk of acute kidney injury regardless of vancomycin trough concentration. J Chemother. 2020; 1-3.

PubMed: https://pubmed.ncbi.nlm.nih.gov/33222657/

28. Miyai T, Imai S, Kashiwagi H, Sato Y, Kadomura S, et al. A Risk Prediction Flowchart of Vancomycin-Induced Acute Kidney Injury to Use When Starting Vancomycin Administration: A Multicenter Retrospective Study. Antibiotics (Basel). 2020; 9: 920. PubMed: https://pubmed.ncbi.nlm.nih.gov/33352848/ 